\title{
PARADIGMAS E EVIDÊNCIAS DA NUTRIÇÃO PERI-OPERATÓRIA
}

\author{
PARADIGMS AND EVIDENCE OF PERIOPERATIVE NUTRITION
}

\author{
Maria Isabel Toulson Davisson Correia, TCBC-MG'; Rodrigo Gomes da Silva, ACBC-MG ${ }^{2}$
}

\section{Introdução}

A abordagem nutricional de pacientes cirúrgicos é aspecto fundamental no cuidado dos mesmos. Múltiplos estudos randomizados, meta-análises e revisões têm abordado o tema. No entanto, alguns aspectos ainda continuam a ser alvo de inúmeras controvérsias, especialmente no tocante ao período adequado de jejum pré-operatório e à liberação da dieta no pós-operatório. Esses aspectos envolvem alguns dos paradigmas existentes em Medicina, mais propriamente em Cirurgia, que geram, por sua vez, angústia e medo aos profissionais envolvidos no tratamento de pacientes operados.

Operações eletivas são, em geral, realizadas após 10 a 16 horas de jejum. A rotina é manter-se o paciente sem comer desde a noite anterior ao ato operatório. Esse período é tido como fundamental para que no momento da indução anestésica o estômago esteja completamente vazio e o risco de aspiração seja mínimo. No entanto, esse tempo é suficientemente longo do ponto de vista metabólico, levando a depleção do estoque de glicogênio, o que tem impacto na resposta orgânica ao estresse, além de gerar desconforto a vários pacientes ${ }^{1,2}$. A resposta orgânica apresenta-se aumentada em pacientes submetidos a jejum noturno, quando comparada a pacientes que receberam infusão de carboidratos $^{1,2}$. Logo, o jejum pré-operatório aumenta o estresse metabólico induzido pelo tratamento cirúrgico.

A liberação da dieta no pós-operatório é outro ponto controverso que tem gerado inúmeras discussões. Em geral, os cirurgiões aguardam a resolução do chamado íleo pósoperatório, ou seja, eliminação de flatos ou evacuação, para a liberação da dieta. Isso ocorre por volta do terceiro ou quarto dia pós-operatório. Além disso, a tradição cirúrgica orienta a oferta escalonada ou progressiva da dieta líquida, pastosa até à sólida. Essa prática não só pode contribuir com a piora do estado nutricional de pacientes previamente desnutridos, como aumenta o tempo de internação hospitalar ${ }^{3}$. Por outro lado, pacientes submetidos a grandes intervenções e alimentados precocemente apresentam melhor oxigenação da mucosa intestinal $^{4}$, diminuição da resposta orgânica e do número de complicações no pós-operatório ${ }^{5-7}$, assim como redução do tempo de dismotilidade intestinal ${ }^{8,9}$.
É nosso objetivo nesta revisão abordar o impacto do jejum pré-operatório e da nutrição precoce no pós-operatório, do ponto de vista metabólico e da associação com complicações, tempo de internação, custos e conforto do paciente. Assim sendo, paradigmas que até hoje são a rotina cirúrgica pré e pós-operatória serão, seguramente, colocados em questionamento.

\section{Jejum pré e pós operatório versus resposta orgânica ao estresse}

A necessidade de jejum prolongado, desde a noite que antecede a operação, tem sido questionada e, novas condutas têm sido adotadas ${ }^{10-12}$. Na verdade, essa prática foi iniciada, quando as técnicas anestésicas ainda eram rudimentares o que aumentava significativamente a possibilidade de vômitos e conseqüentemente, o risco de aspiração. Por volta de 1980, quando se começou a questionar o jejum rígido e prolongado, no pré-operatório, já se sabia que o esvaziamento gástrico de água e de outros líquidos com baixo teor calórico (Líquidos claros. Ex. Chá, café e sucos sem polpa), quando avaliado em voluntários sadios, apresentava-se como uma curva exponencial, com queda extremamente abrupta ${ }^{13}$.

No entanto, o grande desafio foi avaliar se os resultados obtidos com voluntários se aplicariam a pacientes sob estresse e ansiedade pré-operatórios. Verificou-se pois, que nem a medicação ansiolítica pré-operatória, nem o nível de ansiedade interferiram no esvaziamento gástrico de líquidos claros e, na acidez gástrica ${ }^{14}$. A regurgitação passiva e a aspiração pulmonar como complicações da anestesia parecem ocorrer apenas quando determinado volume gástrico está presente, provavelmente algo superior a 200 mililitros $^{14}$.

Se a ingestão de líquidos claros no pré-operatório não gerou aumento de complicações pós-operatórias, por outro lado o jejum prolongado no pré-operatório está associado a potencial agravante da resposta orgânica ao estresse. Esta resposta é um fenômeno fisiológico, desencadeado por múltiplos estímulos que atingem o hipotálamo e estimulam o sistema nervoso simpático e a medula supra-renal a liberarem substâncias desencadeadoras da resposta, no intuito de manter a homeostase corporal. No entanto, na presença de estí-

\footnotetext{
1. Professora Adjunta de Cirurgia da Universidade Federal de Minas Gerais; Coordenadora do Grupo de Nutrição do Instituto Alfa de Gastroenterologia, Hospital das Clínicas da UFMG.

2. Professor Adjunto de Cirurgia da Universidade Federal de Minas Gerais. Coordenador do Grupo de Parede e membro do grupo de Coloproctologia do Instituto Alfa de Gastroenterologia, Hospital das Clínicas da UFMG.
}

Recebido em 10/10/2005

Aceito para publicação em 01/12/2005

Conflito de interesse: nenhum

Fonte de financiamento: nenhuma

Trabalho realizado no Instituto Alfa de Gastroenterologia do Hospital das Clínicas da UFMG. 
mulos prolongados e de grande intensidade, a resposta ao estresse torna-se exacerbada, aumentando a morbimortalidade. Além disso, a produção de citoquinas, principalmente de interleucina 1 (IL-1), interleucina 6 (IL-6) e fator de necrose tumoral (FNT), desencadeada pela lesão tecidual, provoca alterações metabólicas importantes e parece estar associada a aumento da resistência periférica à insulina ${ }^{15}$.

Com o advento das técnicas cirúrgicas minimamente invasivas, como a cirurgia videoendoscópica, a magnitude da resposta orgânica pós-cirúrgica foi reduzida. Contudo, pacientes submetidos a grandes intervenções do trato digestivo, em geral para tratamento de doenças neoplásicas e, aqueles vítimas de trauma, ainda sofrem de estresse muito aumentado. No caso de pacientes com câncer, existe ainda o agravante de que via de regra, são doentes em condições nutricionais precárias, grande parte deles desnutridos graves ${ }^{16}$ e que por conseguinte não dispõem de reservas metabólicas.

A oferta de nutrientes no pré-operatório imediato, mais especificamente carbohidratos, tem sido vista como um dos possíveis fatores benéficos, com potencial de minimizar a resposta orgânica. Três estudos recentes, dois em pacientes submetidos a operações ortopédicas ${ }^{17,18}$ e outro, envolvendo pacientes com enfermidades gastrointestinais ${ }^{19}$ mostraram que aqueles pacientes que receberam carbohidratos, no préoperatório, tiveram menor resistência hepática à insulina e diminuição das perdas de nitrogênio, do primeiro ao terceiro dia pós-operatório. Além disso, houve melhor controle glicêmico do grupo tratado, o que pode ser traduzido em menor risco de complicações relacionadas à hiperglicemia.

A hipótese de que a oferta de carbohidratos interfira com a resposta orgânica, conforme citado anteriormente, advém de pesquisas em ratos submetidos a estresse importante. Nesses estudos animais submetidos a grandes estresses, tais como choque hemorrágico ou endotoxemia e, em jejum por seis a 24 horas, apresentaram capacidade inferior de tolerar a agressão quando comparados aos animais que receberam carbohidratos ${ }^{20}$. Os animais alimentados responderam ao trauma com resposta orgânica diminuída, com melhor tolerância à glicose, com força muscular aumentada e menor translocação bacteriana. Após essas observações, buscouse verificar se o mesmo acontecia em seres humanos. A hipótese levantada foi de que a oferta de carbohidratos no préoperatório incorreria em resposta orgânica diminuída. Desta forma, pacientes submetidos à colecistectomia convencional receberam, na noite anterior à operação, $5 \mathrm{mg} / \mathrm{Kg} / \mathrm{minuto}$ por via endovenosa o que resultou em hiperinsulinemia, semelhante ao estado pós-prandial. Os pacientes tiveram redução de $50 \%$ de resistência periférica à insulina no pós-operatório, quando comparados a pacientes em jejum por 12 horas $^{21}$. No entanto, a oferta de carbohidratos por via venosa demanda internação no dia anterior o que gera custos aumentados. Logo, a possibilidade de oferecer a mesma quantidade de carbohidratos por via oral foi avaliada, de modo que os pacientes pudessem ser preparados em suas residências. Foi elaborada bebida rica em carbohidratos contendo $12,5 \%$ de glicose. A oferta dessa bebida na noite anterior à operação (800 ml) e até duas horas antes da indução anestésica (400 ml), em pacientes submetidos a operação colorretal ${ }^{2}$ e colocação de prótese total de quadril ${ }^{22}$ também diminuiu a resistência periférica à insulina.

Por que a resistência periférica à insulina é fator relevante do ponto de vista clínico? A resistência à insulina é fenômeno transitório que dura aproximadamente até três semanas após a realização de operações abdominais eletivas e não complicadas. Na verdade, esse estado metabólico muito se assemelha ao do diabetes mellitus tipo II. Ou seja, a captação de glicose pelas células está diminuída devido à incapacidade do transportador GLUT-4 realizar essa ação e, conseqüentemente, a produção de glicogênio é diminuída. Simultaneamente, há aumento da produção endógena de glicose, por neoglicogênese, de modo que a glicemia sangüínea encontrase elevada o que é sério fator de risco para maior morbimortalidade ${ }^{23}$. Além disso, quanto maior a resistência à insulina maior o tempo de internação ${ }^{16}$.

Logo, com base nas alterações metabólicas induzidas pelo jejum prolongado antes do ato operatório, é de grande importância tentar reverter essa situação. A oferta de líquidos claros não interfere nesse mecanismo. Por outro lado, a bebida rica em carbohidratos mostrou-se efetiva nessa ação, assim como proporcionou diminuição de sede e fome, melhor bem estar e redução da ansiedade e estresse, sem causar aumento de estase gástrica ${ }^{24}$.

A oferta de nutrição precoce no pós-operatório também parece contribuir para a diminuição do estresse cirúrgico ${ }^{6,25}$. Alguns estudos têm demonstrado que o uso de nutrição enteral precoce (até 48 horas, no pós-operatório) melhora a síntese de albumina e globulina, diminui o nível de excreção urinária de catecolaminas e acelera a cicatrização de feridas, entre outros achados ${ }^{6,26,27}$.

Pacientes vítimas de queimaduras quando submetidos à nutrição enteral precoce (quatro horas após o acidente) versus pacientes nutridos no terceiro dia, apresentaram balanço nitrogenado menos negativo já na primeira semana, assim como, diminuição dos níveis de catecolaminas urinárias e de glucagon plasmático ${ }^{28}$. Mais recentemente, Brodner et al ${ }^{27}$ ofereceram dieta oral no primeiro dia pós-operatório a pacientes submetidos a cistectomia radical e compararam com dois outros grupos submetidos tanto a jejum, como a nutrição parenteral. Os autores observaram que o grupo de estudo apresentou menores níveis de catecolaminas plasmáticas e urinárias, assim como balanço nitrogenado menos negativo.

\section{Dieta no pós-operatório e "íleo adinâmico"}

A liberação da dieta e o tipo de alimentos permitidos (líquidos claros progredindo até alimentos sólidos) após a resolução do "íleo" não têm sido apoiados pela luz da Medicina baseada em evidências. Aliás, os primeiros questionamentos começaram a surgir perante o uso rotineiro de cateteres nasogástricos $(\mathrm{CNG})$ no pós-operatório de operações do aparelho digestivo para tratar o chamado íleo adinâmico. Entre outros estudos, o realizado no nosso departamento em $1992^{29}$, mostrou que o uso profilático rotineiro de cateteres nasogástricos não só foi desnecessário como esteve associado a aumento de complicações, principalmente pulmonares e, tempo de internação prolongado. 
Uma meta-análise mostrou que, para cada paciente que utilizou cateter nasogástrico no pós-operatório, pelo menos 20 doentes não demandaram a sua inserção, concluindo, por conseguinte, que o uso rotineiro de CNG não tem base científica para a sua colocação como profilaxia de íleo ${ }^{30}$.

O jejum pós-operatório faz parte dos cuidados de rotina prescritos até que haja eliminação de flatos ou de fezes. Isso, em geral, ocorre em torno do terceiro ou quarto dia pós-operatório. Logo, o tempo de internação hospitalar é maior, pois se aguarda que o doente coma e tolere a dieta para se dar a alta. Após operações colorretais, por exemplo, os pacientes permanecem no hospital por tempo médio de seis a 12 dias $^{31}$. Acredita-se que a dieta precoce possa levar à distensão abdominal, náuseas e vômitos, piorando o "íleo adinâmico". Casto et a ${ }^{\beta 2}$ definiram essa prática como "antiquada e existente há mais de 100 anos, quando a incidência de vômitos pós-operatórios era muito mais elevada devido ao uso de anestésicos rudimentares".

Alguns autores têm estudado o impacto da dieta precoce na recuperação pós-operatória e todos esses paradigmas têm sido desafiados e questionados. Alguns estudos têm mostrado que após a realização de operações colorretais é possível alimentar os doentes precocemente e dar alta no $2^{\circ}$. e $3^{\circ}$. dias pós-operatórios ${ }^{33}$. Essa prática diminui o desconforto dos doentes, o tempo de internação e conseqüentemente, os custos hospitalares.

Porque deveremos esperar pela eliminação de flatos ou fezes para começar a alimentar os pacientes, no pós-operatório? Até ao momento, duas plausíveis justificativas eram usadas. A primeira, é a crença de que se deva aguardar pela resolução da dismotilidade intestinal transitória (íleo pós-operatório). A segunda é justificada pela possibilidade de que a alimentação precoce aumente o risco de fístulas anastomóticas. Este aspecto não mais é validado pelas evidências da atualidade. A cicatrização das anastomoses e as complicações pósoperatórias são diretamente afetadas por diversos fatores tais como: estado nutricional do doente ${ }^{34}$; uso de drogas imunossupressoras como corticóides; condições locais do abdômen influenciadas pela presença de inflamação, infecção, e/ou câncer; fluxo esplâncnico adequado e; boa técnica cirúrgica, entre outros ${ }^{35}$. Por outro lado, sabe-se que a nutrição precoce melhora a cicatrização e o fluxo esplâncnico, estimula a motilidade intestinal, conseqüentemente diminuindo a estase e, reduz a incidência de morbimortalidade ${ }^{4,36,37}$. Em estudo prospectivo randomizado realizado por Aguilar-Nascimento et $a l^{38}$, verificou-se que não houve diferença significante entre as taxas de deiscência de anastomose entre o grupo que recebeu nutrição precoce e o submetido a tratamento convencional. A nutrição precoce é viável e segura, mesmo quando oferecida entre quatro a 12 horas após a operação ${ }^{34-41}$. Na verdade, a oferta precoce de nutrientes estimula reflexo que produz atividade propulsiva coordenada e, induz à secreção de hormônios gastrointestinais, diminuindo por conseguinte o íleo pós-operatório. Anderson et al ${ }^{42}$ mostraram, em pacientes submetidos a operações colorretais, que o grupo que recebeu tratamento envolvendo múltiplas estratégias, no qual a nutrição precoce era uma das variáveis, apresentou recuperação mais rápida e melhora da função gastrointestinal, cuja média foi de 48 horas (33-55 h) versus 76 horas (70-110h) no grupo controle.
Até o momento, o tratamento convencional adotado pela maior parte dos hospitais e clínicas, recomenda o jejum em conjunto com a administração de líquidos intravenosos até que haja eliminação de flatos ou fezes. Na verdade, não existem protocolos que orientem sobre a liberação da dieta na maioria dos centros. Por exemplo, no Reino Unido, 78,5\% das unidades obstétricas questionadas informaram que a decisão de alimentar as pacientes após a realização de cesarianas é feita sem nenhuma rotina estabelecida ${ }^{43}$.

A etiologia do íleo pós-operatório é multi-fatorial. Alterações do sistema nervoso autônomo, de neurotransmissores, de fatores inflamatórios locais e da resposta metabólica/inflamatória assim como, a presença de diferentes hormônios, a anestesia e a analgesia pós-operatória têm sido descritos como fatores causais. De sorte que uma abordagem multivariada deve ser usada para minimizar ou tratar a disfunção fisiológica da motilidade após procedimentos cirúrgicos ${ }^{8,44-47}$.

A oferta de nutrição precoce é uma dessas variáveis que deve ser associada à anestesia epidural, cirurgia minimamente invasiva, medicações antieméticas, mobilização precoce no pós-operatório e preparo psicológico do paciente, entre outras (Tabela 1). Na verdade, os estudos que não associaram a nutrição precoce aos outros procedimentos usados para reduzir a dismotilidade intestinal não mostraram benefícios destes na evolução pós-operatória. Stewart et $a l^{48}$, em estudo prospectivo randomizado com pacientes submetidos a ressecção colônica que receberam líquidos à vontade, dentro das primeiras quatro horas de pós-operatório, verificaram que a tolerância foi boa. A maioria dos pacientes (80\%) assim tratada tolerou uma refeição habitual dentro das primeiras 48 horas. As taxas de vômitos, recolocação de CNG e distensão abdominal não foram significantemente diferentes entre esse grupo e o controle.

Os pacientes idosos são mais suscetíveis a complicações e sabidamente permanecem internados por mais tempo. Estudos recentes, avaliaram o comportamento de pacientes idosos quando tratados com estratégias multivariadas e, diferentes resultados foram descritos. Delaney et al ${ }^{49}$ não conseguiram mostrar vantagens desse tipo de abordagem em pacientes com idade superior a 70 anos. Os autores não verificaram diminuição do tempo de internação no grupo de idosos assim tratado, porém numa análise detalhada de pacientes mais jovens, o tempo de internação foi reduzido de 7,1 dias para cinco dias. Por outro lado, Di Fronzo et l $^{8}$ observaram pacientes com idades acima de 70 anos e relataram que $89,6 \%$ deles toleraram a dieta precoce muito bem, tendo permanecido internados em média por 3,9 dias.

Uma meta-análise recente registrou diminuição do tempo de internação em 0,84 dias $(0,36-1,33 \text { dias })^{6}$.

É razoável esperar-se que a abordagem multivariada resulte em menor tempo de internação. No entanto, várias modalidades de tratamento e diversas variáveis têm sido utilizadas, o que seguramente tem contribuído para os diferentes resultados. É difícil comparar esses resultados frente às múltiplas possibilidades relacionadas ao tema. Salientam-se: diferentes definições de dismotilidade pós-operatória; estudos com tamanhos de amostra insuficientes; diferenças nas operações realizadas; múltiplos protocolos anestésicos; várias co-morbidades dos pacientes e, por último, diversas variáveis-resposta, tais como passagem de 
flatos e fezes, movimentos peristálticos, alta hospitalar e tolerância a alimentos sólidos. Porém, existe evidência clara dos benefícios em se usar abordagem multivariada para melhorar as alterações pós-operatórias, o que diretamente impacta no tempo de internação e nos custos.

A liberação progressiva de nutrientes, iniciada com líquidos claros até alimentos sólidos, tem sido outro aspecto controverso e discutido por muitos cirurgiões que na sua maioria ainda acreditam que essa seqüência deva ser respeitada. Contudo, em estudo prospectivo randomizado realizado no nosso serviço, Sanches et al $l^{51}$ acompanharam 165 doentes submetidos a tratamento cirúrgico eletivo para afecções gastrointestinais. A um grupo foi-lhe permitido ingerir dieta livre tão logo esta foi liberada e o outro grupo, recebeu alimentos de acordo com a progressão habitual (líquidos claros até dieta regular). Os autores não encontraram nenhuma diferença entre os dois grupos, no tocante à incidência de complicações ou à intolerância à dieta. Os pacientes que ingeriram a dieta livre receberam mais calorias no primeiro dia da dieta $(917,13$ calorias versus 467,94 calorias). Os autores recomendam a liberação de dieta livre como primeira opção tão logo esta seja liberada. Em outro estudo, Jeffery et $a^{52}$ comparando os dois tipos de dieta, líquida restrita versus livre, verificaram que a incidência de complicações foi semelhante entre os dois grupos (7,5\% versus $8,1 \%)$. Segundo os autores, este tipo de conduta é bem tolerado, apresenta-se mais palatável para o doente, é mais fácil de engolir e diminui o tempo de hospitalização.

Novas perspectivas sobre ações que possam ter impacto sobre o íleo pós-operatório devem ser avaliadas no intuito de minimizar os efeitos deletérios do mesmo e todas as facetas a ele relacionadas. Recentemente, foi relatada que a simples mastigação de bala de goma no pós-operatório de pacientes submetidos a colectomia vídeoendoscópica foi capaz de reduzir o íleo pós-operatório ${ }^{43}$. O grupo estimulado a mastigar as balas de goma três vezes ao dia, desde o primeiro dia pós-operatório, apresentou eliminação de flatos em média no segundo dia versus no terceiro dia, no grupo controle. A primeira eliminação de fezes ocorreu em 2,7 dias versus 5,8 dias de pós-operatório e o tempo de internação foi de 13,5+/3,0 dias contra 14,5+/-6,1 dias. Os autores concluíram que a tolerância a essa opção de tratamento foi boa e que é método não dispendioso para estimular o retorno da atividade motora intestinal.

\section{Conclusão}

A resposta orgânica ao estresse e o íleo pós-operatório continuam a ser realidades que podem interferir de maneira significativa na evolução dos pacientes, de acordo com a sua magnitude e duração. Ambos representam resposta fisiológica que, quando perpetuada, traduz-se em aumento de morbimortalidade. A sua etiologia é multi-fatorial e é melhor tratada com a combinação de diversas abordagens, das quais a nutrição tanto no pré como no pós-operatório tem papel relevante. Na medida que a ciência se desenvolve, é importante que antigos paradigmas, muitos deles baseados em condutas empíricas, sejam questionados com a realização de estudos clínicos randomizados. Quando diferentes resultados são encontrados, novas estratégias devem ser testadas e seguidas, objetivando a melhora do tratamento dos pacientes e, concomitantemente, a redução de custos.

Thomas Kuhn ${ }^{53}$, físico americano, autor de "As estruturas das Revoluções Científicas" disse: "A ciência não é uma transição suave do erro à verdade, e sim uma série de crises ou revoluções, expressas como mudanças de paradigmas."

Tabela 1 - Abordagem multivariada.

$\overline{\text { Avanços recentes, em pacientes submetidos a tratamento }}$ cirúrgico.

- Preparo psicológico do paciente em relação ao que irá enfrentar no pós-operatório

- Oferta de carbohidratos, por via oral, no pré-operatório até duas horas antes da operação

- Anestesia epidural

- Cirurgia minimamente invasiva

- Medicações antieméticas no per e pós-operatório

- Analgesia adequada, se possível, pelo cateter epidural

- Nutrição precoce

Mobilização precoce no pós-operatório

\begin{abstract}
Understanding perioperative pathophysiology and implementing care regimes, through a multimodal approach, to reduce the organic response to stress after surgery and the related postoperative ileus, are major challenges. Multimodal surgical strategies such as pre-operative intake of a carbohydrate drink, instead of the usually recommended 2- to 6-hour period of nothing-bymouth, together with patient's education of the postoperative care plan, plus efficacious analgesia and early postoperative nutrition, among others, have been described to significantly impact on the previous variables. Therefore, these strategies accelerate rehabilitation and, as a consequence, decrease complications and hospital length of stay and, its related costs (Rev. Col. Bras. Cir. 2005; 32(5): 342-347).
\end{abstract}

Key words: Nutrition; Ileum; Postoperative period; Analgesia; Diet.

\title{
REFERÊNCIAS
}

1. Ljungqvist O, Thorell A, Gutniak M, et al. Glucose infusion instead of preoperative fasting reduces postoperative insulin resistance. J Am Coll Surg. 1994;178(4):329-36.
2. Nygren J, Soop M, Thorell A, et al. Preoperative oral carbohydrate administration reduces postoperative insulin resistance. Clin Nutr.1998;17(2):65-71. 
3. Di Fronzo LA, Yamin N, Patel K, et al. Benefits of early feeding and early hospital discharge in elderly patients undergoing open colon resection. J Am Coll Surg. 2003;197(5):747-52.

4. Braga M, Gianotti L, Gentilini O, et al. Feeding the gut early after digestive surgery: results of a nine-year experience. Clin Nutr. 2002;21(1):59-65.

5. Enzi G, Casadei A, Sergi G, et al. Metabolic and hormonal effects of early nutritional supplementation after surgery in burn patients. Crit Care Med. 1990; 18(7):719-21.

6. Kudsk KA. Gut mucosal nutritional support-enteral nutrition as primary therapy after multiple system trauma. Gut. 1994;35(1 Suppl):S52-4.

7. Moore FA, Feliciano DV, Andrassy RJ, et al. Early enteral feeding, compared with parenteral, reduces postoperative septic complications. The results of a meta-analysis. Ann Surg. 1992;216(2):172-83.

8. Di Fronzo LA, Cymerman J, O'Connell TX. Factors affecting early postoperative feeding following elective open colon resection. Arch Surg. 1999;134(9):941-5; discussion 945-6.

9. Waldhausen JH, Shaffrey ME, Skenderis BS, et al. Gastrointestinal myoelectric and clinical patterns of recovery after laparotomy. Ann Surg. 1990;211(6):777-84; discussion 785.

10. Practice guidelines for preoperative fasting and the use of pharmacological agents to reduce the risk of pulmonary aspiration: application to healthy patients undergoing elective procedures: a report by the American Society of Anaesthesiologists Task Force on Preoperative Fasting. Anesthesiology. 1999;90(3):896-905.

11. Erskine L, Hunt JN. The gastric emptying of small volumes given in quick succession. J Physiol. 1981;313:335-41.

12. Nygren J, Thorell Am, Ljungqvist O. New developments facilitating nutritional intake after gastrointeestinal surgery. Curr Opin Clin Nutr Metab Care. 2003;6(5):593-7.

13. Brener W, Hendrix TR, McHugh PR. Regulation of the gastric emptying of glucose. Gastroenterology. 1983;85(1):76-82.

14. Haavik PE, Soreide E, Hofstad B, et al. Does preoperative anxiety influence gastric fluid volume and acidity? Anesth Analg. 1992;75(1):9-4.

15. Thorell A, Nygren J, Ljungqvist O. Insulin resistance: a marker of surgical stress. Curr Opin Clin Nutr Metab Care 1999;2(1):6978.

16. Correia, MI, Caiaffa WT, da Silva AL, et al. Risk factors for malnutrition in patients undergoing gastroenterological and hernia surgery: an analysis of 374 patients. Nutr Hosp. 2001;16(2):5964.

17. Soop M, Nygren J, Mammarqvist F, et al. Preoperative oral carbohydrate treatment attenuates postoperative whole body nitrogen losses and hepatic insulin resistance. Clin Nutr. 2001;20(suppl 1):50.

18. Soop M, Nygren J, Thorell A, et al. Preoperative oral carbohydrate treatment attenuates endogenous glucose release 3 days after surgery. Clin Nutr. 2004;23(4):733-41.

19. Henriksen MG, Hessov I, Dela F, et al. Effects of preoperative oral carbohydrates and peptides on postoperative endocrine response, mobilization, nutrition and muscle function in abdominal surgery. Acta Anaesthesiol Scand. 2003;47(2):191-9.

20. Ljungqvist O, Nygren J, Thorll A. Preoperative nutrition therapy - novel developments. Scan J Nutr. 2000;44:3-7.

21. Ljungqvist O, Thorell A, Gutniak M, et al. Glucose infusion instead of preoperative fasting reduces postoperative insulin resistance. J Am Coll Surg. 1994;178(4):329-36.

22. Soop M, Nygren J, Myrenfors P, et al. Preoperative oral carbohydrate treatment attenuates immediate postoperative insulin resistance. Am J Physiol Endrinol Metab. 2001;280: E576-83.
23. van den Berghe $G$, Wouters $P$, Weekers $F$, et al. Intensive insulin therapy in the critically ill patients. N Engl J Med. 2001;345(19):1359-67.

24. Ljungqvist O, Soreide E. Preoperative fasting. Br J Surg. 2003;90(4):400-6.

25. Moss G, Koblenz G. Postoperative positive nitrogen balance: effect on wound and plasma protein synthesis. Surg Forum. 1970;21:71-3.

26. Brodner G, Van Aken H, Hertle L, et al. Multimodal perioperative management - combining thoracic epidural analgesia, forced mobilization, and oral nutrition - reduces hormonal and metabolic stress and improves convalescencec after major urologic surgery. Anesth Analg. 2001;92(6):1594-600.

27. Gianotti L, Nelson JL, Alexander JW, et al. Post injury hypermetabolic response and magnitude of bacterial translocation: prevention by early enteral nutrition. Nutrition. 1994;10(3): 225-31.

28. Chiarelli A, Enzi G, Casadei A, et al. Very early nutrition supplementation in burned patients. Am J Clin Nutr. 1990;51(6):1035-9.

29. Savassi-Rocha PR, Conceição SA, Ferreira JT, et al. Evaluation of the routine use of the nasogastric tube in digestive operation by a prospective controlled study. Surg Gynecol Obstet. 1992;174(4):317-20.

30. Cheatham ML, Chapman WC, Key SP, et al. A meta-analysis of selective versus routine nasogastric decompression after elective laparotomy. Ann Surg. 1995;221(5):469-76

31. Basse L, Billesbolle P, Jakobsen DH, et al. A clinical pathway to accelerate recovery after colonic resection. Ann Surg. 2000;232(1):51-7.

32. Johnson Casto C, Krammer J, Drake J. Postoperative feeding: a clinical review. Obstet Gynecol Surv. 2000;55(9):571-3.

33. Basse L, Thorbol JE, Lossl K, et al. Colonic surgery with accelerated rehabilitation or conventional care. Dis Colon Rectum. 2004;47(3):271-7; discussion 277-8.

34. Correia MITD, Waitzberg DL. The impact of malnutrition on morbidity, mortality, length of hospital stay and costs evaluated through a multivariate model analysis. Clin Nutr. 2003;22(3):235-9.

35. Campos AC, Andrade DF, Campos GM, et al. A multivariate model to determine prognostic factors in gastrointestinal fistulas. J Am Coll Surg. 1999; 188(5):483-90.

36. Di Fronzo LA, Cymerman J, O'Connell TX. Factors affecting early postoperative feeding following elective open colon resection. Arch Surg.1999;134(9):941-5; discussion 945-6.

37. Enzi A, Casadei G, Sergi G, et al. Metabolic and hormonal effects of early nutritional supplementation after surgery in burn patients. Crit Care Med. 1990; 18(7):719-21.

38. Aguilar-Nascimento JE, Goelzer J. Alimentação precoce após anastomoses intestinais: riscos ou benefícios? Rev Assoc Med Bras. 2002;48(3):348-52.

39. Asao T, Kuwano H, Nakamura J, et al. Gum chewing enhances early recovery from postoperative ileus after laparoscopic colectomy. J Am Coll Surg. 2002;195(1):30-2.

40. Reissman P, Teoh TA, Cohen SM, et al. Is early oral feeding safe after elective colorectal surgery? A prospective randomized trial. Ann Surg. 1995;222(1):73-7.

41. Senkal M, Haaker R, Deska T, et al. Early enteral gut feeding with conditionally indispensable pharmaconutrients is metabolically safe and is well tolerated in postoperative cancer patients - a pilot study. Clin Nutr. 2004;23(5):1193-8.

42. Anderson ADG, McNaught CE, MacFie J, et al. Randomized clinical trial of multimodal optimization and standard perioperative surgical care. Br J Surg. 2003;90(12):1497-504. 
43. Worthington LM, Mulcahy AJ, White S, et al. Attitudes to oral feeding following caesarean section. Anaesthesia.1999;54(3):292-6.

44. Livingston EH, Passaro EP. Postoperative ileus. Dig Dis Sci. 1990;35(1):121-31.

45. Resnick J, Greenwald DA, Brandt LJ. Delayed gastric emptying and postoperative ileus after nongastric abdominal surgery: part I. Am J Gastroenterol. 1997;92(5):751-62.

46. Henriksen MG, Hansen HV, Hessov I. Early oral nutrition after elective colorectal surgery: influence of balanced analgesia and enforced mobilization. Nutrition. 2002;18(3):263-7.

47. Correia MI, da Silva RG. The impact of early nutrition on metabolic response and postoperative ileus. Curr Opin Clin Nutr Metab Care. 2004;7(5):577-83.

48. Stewart BT, Woods RJ, Collopy BT, et al. Early feeding after elective open colorectal resections: a prospective randomized trial. Aust N Z J Surg. 1998;68(2):125-8.

49. Delaney CP, Zutshi M, Senagore AJ, et al. Prospective, randomized, controlled trial between a pathway of controlled rehabilitation with early ambulation and diet and traditional postoperative care after laparotomy and intestinal resection. Dis Colon Rectum. 2003;46(7):851-9.
50. Lewis SJ, Egger M, Sylvester PA, et al. Early enteral feeding versus "nil by mouth" after gastrointestinal surgery: systematic review and meta-analysis of controlled trials. BMJ. 2001;323(7316):773-6.

51. Sanches MD, Castro L P, Sales TR, et al. Comparative study about progressive versus free oral diet in postoperative period of digestive surgeries. Gastroenterology 1996;110(1):37-38.

52. Jeffery KM, Harkins B, Cresci G, et al. The clear liquid diet is no longer a necessity in the routine postoperative management of surgical patients. Am Surg. 1996;62(3):167-70.

53. Kuhn TS. The Structure of Scientific Revolutions. Chicago: University of Chicago Press; 1962.

Endereço para correspondência:

Maria Isabel Toulson Davisson Correia

Avenida Carandaí 246 apt. 902

Belo Horizonte, MG, 30130-060

isabel_correia@uol.com.br

Telefones: 31 91688239; 3132712661 